1

2

3

4

5

6

7

8

9

10

\title{
Effects of smoking on healing response to non-surgical periodontal therapy: A multilevel modeling analysis
}

Chi Pui Wan, W. Keung Leung*, May C. M. Wong, Ruby M. S. Wong, Peng Wan,

Edward C. M. Lo, Esmonde F. Corbet

Faculty of Dentistry, The University of Hong Kong, Hong Kong SAR, China

Running title: smokers' healing: multilevel analysis

Keywords: Periodontitis; Smoking; Models, Statistical;

*Corresponding author:

W. K. Leung, Room 3B39, 34 Hospital Road, Prince Philip Dental Hospital, Faculty of Dentistry, The University of Hong Kong, Hong Kong SAR, China. Telephone:

+852-28590417; Fax: +852-28587874; E-mail: ewkleung@ @kucc.hku.hk

Date of resubmission: November 21, 2008 


\section{Conflict of interest and sources of funding statement}

The authors declare that they have no conflict of interests.

The work described in this paper was partially supported by grants from the Research

Grants Council of the Hong Kong Special Administrative Region, China (HKU 7331/00M) and Merck Sharp \& Dohme (Asia) Limited (26004077). 
Wan CP, Leung WK, Wong MCM, Wong RMS, Wan P, Lo ECM, Corbet EF. Effects of smoking on healing response to non-surgical periodontal therapy: A multilevel modeling analysis. J Clin Periodontol

Abstract

Aim: To investigate factors predicting non-surgical periodontal treatment responses using multilevel multiple regression.

Methods: 40 men (mean 45.6 years) were recruited. 20 were smokers. 12-month reduction in probing pocket depth (PPD) and gain in probing attachment level (PAL) of 5814 sites were analyzed with 594 being initially diseased sites (initial PPD $\geq$ $5 \mathrm{~mm})$.

Results: Variance Component models showed site level variations contributed about $70-90 \%$ of the total variance. About $10 \%$ reduction of the total variations of PPD reduction in initially diseased sites was achieved with the inclusion of the 10 predictors in the multilevel multiple regression. Multilevel multiple regression showed that three predictors - subject-level: non-smokers; tooth-level: anterior teeth; site-level: sites without plaque at baseline, were significantly associated with greater reduction in PPD in initially diseased sites over the 12 months study period. $(\mathbf{p}<0.05)$. No consistent predictor was found for PAL gain.

Conclusion: Multilevel analysis was applied on periodontal treatment response data. Smokers showed less favorable PPD reduction at deep sites after non-surgical 
periodontal therapy. 
Smoking is considered as a well-established risk factor for periodontal diseases, a chronic infectious disorder caused by bacterial plaque characterized by destruction of tooth supporting tissue. Smokers have increased risks of experiencing periodontal attachment loss (Grossi et al. 1994, Haffajee and Socransky 2001a, Susin et al. 2004, Ng \& Leung, 2006), radiographic bone loss (Grossi et al. 1995, Bergstrom 2004, Baljoon et al. 2005) and tooth loss post-treatment (Leung et al. 2006, Matuliene et al. 2008). Smokers are found to harbor a higher prevalence of periodontal pathogens (Haffajee \& Socransky, 2001b, van Winkelhoff et al. 2001).

Apart from alterations of the periodontal microflora, smoking has been shown to adversely affect the host immune response in various respects, including impaired neutrophil function (Mariggio et al. 2001, Güntsch et al. 2006), lowered immunoglobulin production (Mooney et al. 2001, Apatzidou et al. 2005), reduced fibroblast function (Raulin et al. 1988), altered inflammatory mediator production (Boström et al. 1998, 1999; Giannopoulou et al., 2003) and vasoconstrictive effects of tissue exposed to cigarette smoke (Mirbod et al. 2001).

Non-surgical mechanical periodontal therapy, including oral hygiene instruction, scaling and root planing, is an effective treatment modality for periodontal disease (Van der Weijden \& Timmerman 2002, Sanz \& Teughels, 2008); however, numerous studies have indicated that smokers generally undergo less favorable improvements in response to non-surgical therapy (Preber \& Bergstrom, 1986; Preber et al., 1995; Renvert et al., 1998; Jin et al., 2000). A systematic review evaluating the effect of smoking on non-surgical periodontal therapy (Labriola et al., 2005) found that the mean difference in probing pocket depth (PPD) reduction with an initial probing depth of $5 \mathrm{~mm}$ or more would be $0.433 \mathrm{~mm}$ favoring non-smokers. On the other hand, the same meta-analysis showed that there was no evidence of a difference observable in 
clinical attachment level gain between smokers and non-smokers after non-surgical periodontal therapy, although a review of clinical evidence (Heasman et al. 2006) suggests that the majority of studies do show that smokers gain less clinical attachment gain in response to periodontal therapy. It is agreed that achieving optimal treatment responses to non-surgical periodontal therapy in smokers is a challenging task and that the treatment outcome of the therapy may vary from patient to patient and also vary among different teeth and tooth sites. It would be beneficial to understand factors at patient, tooth and site levels that may affect these variations in treatment response in both smokers and non-smokers.

Since the early 1990s, researchers have questioned the utility of single level statistical analysis of site-level or tooth-level data in periodontal clinical trials because the correlations among sites and/or teeth within subjects invalidates these methods. In applying single level statistical analysis to periodontal data, many earlier publications chose to present average sites' measurements generated on a subject level. However, such an approach may not explicitly reflect the site-specific nature of periodontal disease (Albandar \& Goldstein 1992, Gilthorpe et al. 2000a, Gilthorpe et al. 2000b, Gilthorpe et al. 2001). Application of multilevel modeling analysis, which takes the clustering effect of periodontal research data into consideration, may provide a more accurate explanation of the natural hierarchical structure of clinical findings of periodontitis and the healing responses after periodontal therapy. Two reports lately, adopted such approach in their periodontal trial data analysis (Tomasi et al. 2007, Matuliene et al. 2008).

In the present prospective study, clinical healing responses of two groups of male Chinese subjects: smokers or non-smokers - matched according to age, pre-operative oral hygiene levels and periodontal disease severity - were recorded after non-surgical periodontal therapy. The aim of this study was to compare the 12-month healing 
response of male Chinese smokers and non-smokers with chronic periodontitis after non-surgical mechanical periodontal therapy using multilevel modeling analysis. The clinical data would be analyzed at site level. The null hypothesis of this clinical trial is that there is no difference in healing responses after non-surgical mechanical periodontal therapy of periodontitis affected male Chinese smokers and non-smokers.

\section{Materials and methods}

\section{Sample size determination}

This clinical study targeted subjects with chronic periodontitis who were otherwise systemically healthy. Sample size for the study was computed as follows. In a study among the same local population, patients with chronic periodontitis showed $4.6 \mathrm{~mm}$ of probing pocket depth (PPD) reduction at 12 months after non-surgical therapy, with standard deviation (SD) of $1.6 \mathrm{~mm}$ (Tong et al. 2003). Assuming that the SD would be the same for smokers and an expected difference of PPD reduction at the initially diseased sites between smokers and non-smokers of $2 \mathrm{~mm}, 20$ subjects in each group were required to enable such a difference to be detected.

\section{Patient selection and screening}

New male patients attending the Reception Clinic of the Prince Philip Dental Hospital, Faculty of Dentistry, The University of Hong Kong and satisfying the inclusion criteria were recruited to participate in the study. The target sample size was at least 22 subjects for each group, to allow for retention of 20 subjects in each group at 12 months. For inclusion, patients had to be free of systemic disease, not undergoing orthodontic treatment, and displaying the following features:

1. 35- to 64-years-old ethnic Chinese with untreated chronic periodontitis 
2. Smokers with a smoking habit of $\geq 10$ cigarettes per day for at least 10 years and expressing no interest in quitting smoking in the coming 12 months

3. Non-smokers with a smoking history of never having smoked

4. At least 16 standing teeth, with at least 1 tooth having PPD $\geq 5 \mathrm{~mm}$ in each quadrant, excluding the third molars.

Subjects were excluded if the patient interview revealed:

1. Known systemic diseases

2. History of taking systemic antibiotics in the preceding 30 days

3. History of dental treatment, other than oral hygiene instructions, in the preceding 30 days

The target sample size for each group was secured six months after the commencement of recruitment.

\section{Patient management and non-surgical mechanical periodontal treatment}

The clinical study was carried out in the Periodontology Clinic, Prince Philip Dental Hospital, Faculty of Dentistry, The University of Hong Kong. Emergency treatment such as extraction, caries stabilization, initial endodontic therapy, if necessary, was completed before the non-surgical periodontal treatment. Six tooth-sites (mesio-buccal, mid-buccal, disto-buccal, mesio-lingual, mid-lingual, and disto-lingual) of each standing tooth were included in this study. One member of the research team (W.K.L.) checked the eligibility of all subjects and that all necessary pre-treatment preparations had been carried out. Receptionists of the Periodontology Clinic were then instructed to arrange the non-surgical periodontal treatment appointments (4-6 visits) under local anesthesia for all subjects to be delivered by a group of six 
experienced dental hygienists within an 8-week period. Both smokers and non-smokers received the same non-surgical periodontal treatment, namely oral hygiene instruction regarding brushing and interdental cleaning, followed by quadrant-wise debridement under local anesthesia. Two research group members (P.W. and R.M.S.W.) at the end of the last dental hygienist treatment appointment independently clinically assessed the quality of the hygienists' care to ensure the completeness of the non-surgical periodontal therapy.

Any residual periodontal problems at conclusion of the study at 12 months, namely any sites with residual PPD $\geq 5 \mathrm{~mm}$, were followed-up and appropriate periodontal treatment e.g. re-root planing or surgical treatment was arranged and delivered without delay. Smoking subjects were again reminded of the deleterious effects resulting from their continued smoking.

\section{Clinical examination}

This was a 12-month prospective clinical study. Clinical parameters were obtained from the patients at baseline, and at 3, 6 and 12 months after completion of non-surgical therapy. All clinical examinations were performed by one examiner (C.P.W.).

Probing pocket depth (PPD) and probing attachment level (PAL) were measured and recorded for six sites of each tooth, excluding third molars. Custom-made poly-ethylene occlusal stents were made for each patient as reference guides for reproducibility of probing sites and for measurement of probing attachment level throughout the study. Except for initial baseline PAL data, which was collected using manual periodontal probe (PCP-UNC 15, Hu-Friedy probe ${ }^{\circledR}$, Chicago, IL), each site was probed with an automated controlled-force periodontal probe, Florida Probe ${ }^{\circledR}$ (Florida Probe Co.). Probe tips were $0.45 \mathrm{~mm}$ in diameter and manufactured from 
implant grade titanium. The resolution of $0.2 \mathrm{~mm}$ could be detected with controlled force of 15g. Presence of plaque was recorded dichotomously as presence or absence of plaque according to detection of plaque deposits determined by running the tip of a periodontal probe along the tooth surface at the gingival margin of each site. Bleeding on probing (BOP) was designated as positive if bleeding occurred within 10 seconds after periodontal probing using the electronic probe.

\section{Ethics}

The research protocol was approved by the Ethics Committee, Faculty of Dentistry, The University of Hong Kong. Written informed consent was obtained from all participants before the commencement of the study.

\section{Data analysis}

\section{Routine statistical analysis}

The data collected was entered into a computer and analyzed using the statistical software package (SPSS). For comparing the difference in healing response between smokers and non-smokers at the subject level, the primary efficacy measure was change in PPD and change in PAL and the secondary efficacy measures included $\mathrm{PI} \%, \mathrm{BOP} \%$ and percentage of sites $\geq 5.0 \mathrm{~mm}$. The significance level was set at $p<$ 0.0017 for multiple comparisons at the 3-, 6- and 12-month recalls within groups or between groups. Differences between groups and between different time-points within groups were tested by Mann-Whitney $U$ test and Wilcoxon signed rank test respectively. 


\section{Multilevel analysis}

In order to account for the hierarchical structure of periodontal disease measurements, site measurements clustered around individual teeth and then teeth clustered within subjects, analysis using a multilevel approach was adopted in this study (Gilthorpe et al. 2000b). PPD reductions at site level at 3 months, 6 months and 12 months (compared to baseline PPD) were analyzed using multilevel multiple regressions. A 3-level random intercept regression model was constructed: site at level 1 , tooth at level 2 and subject at level 3. Variance Components models (with no independent variables included) were obtained initially to investigate the variance of the PPD reductions across all the 3 levels. At different levels the random effects were assumed to be uncorrelated and followed normal distributions. Subsequently, ten independent variables with five on the subject-level, two on the tooth-level and three on the site-level were included in the multilevel multiple regression model. The five subject-level variables were: smoking (non-smoker vs. smoker), age (in years), number of missing teeth at baseline, \% sites with plaque at baseline and \% sites with BOP at baseline. The two tooth-level variables considered in the regression model were: the tooth position (posterior [premolars and molars] vs. anterior [incisors and canines]) and arch (lower vs. upper). The three site-level variables were: presence or absence of plaque at baseline, presence or absence of BOP at baseline, and surface (lingual vs. buccal). All the continuous variables were centered (subtracted from the mean) before the analysis. The analyses of the gain in PAL at 3, 6 and 12 months were performed in a similar manner: 3-level regression models were considered with ten independent variables. All the analyses were performed using the software MLwiN 2.1 (Rasbash et al. 2000). The level of significance was set at 0.05 .

In order to focus on the factors affecting the change of PPD and PAL of initially 
diseased sites (sites with PPD $\geq 5.0 \mathrm{~mm}$ at baseline), above-mentioned multilevel multiple regressions were repeated for initially diseased sites only. Again, the level of significance was set to be at 0.05 .

\section{Results}

\section{Routine statistical analysis}

Change of PPD and PAL at all sites

In the present study, 23 non-smokers and 23 smokers were recruited. Forty of the enrolled subjects completed the study, 3 subjects being lost to follow-up in both the smoker and the non-smoker groups. One smoker and three non-smokers could not attend the scheduled recalls due to contemporaneous conflict with their job time-tables. Two smokers quitted smoking, one for personal reasons and the other having been diagnosed to be suffering from hypertension was successfully counseled to quit smoking by his physician.

Mean ages of the smokers and non-smokers who completed the study were $46.2 \pm$ 6.8 and $45.0 \pm 5.9$ years, respectively. Regarding the tobacco consumption of smokers, 6 were light smokers while the remaining 14 were moderate smokers (Grossi et al. 1994). Their smoking-pack-years were $20.8 \pm 8.7$, ranging from 10 to 30 . Mean number of missing teeth (excluding third molars) was $3.9 \pm 2.9$ teeth for smokers and $3.7 \pm 2.8$ teeth for non-smokers $(\mathrm{P}>0.05)$. Other clinical data are shown in Table 1 . There was no difference between non-smokers and smokers in percentage of plaque, mean full-mouth PPD, mean full-mouth PAL and percentage of sites with PPD $\geq 5 \mathrm{~mm}$ at baseline. Both groups showed poor oral hygiene and a high percentage of sites with BOP at baseline, while smokers exhibited significantly less bleeding compared with non-smokers $(p=0.003)$.

Table 1 shows the change of subject level clinical parameters over the study 
period. Throughout the course of the study, both non-smokers and smokers achieved favorable improvements in their plaque control. This was demonstrated by significant reductions of $\mathrm{P} 1 \%$ at 3, 6 and 12 months compared to baseline in both groups. By 12 months, the mean $\mathrm{P} 1 \%$ was reduced to less than $34 \%$.

In addition, in response to non-surgical mechanical periodontal therapy, both groups showed significant reductions in mean full-mouth BOP\% compared to baseline. By 12 months, the mean BOP\% was reduced to less than $27 \%$.

During the 12-month study period, full-mouth mean PPD in both groups was found to be significantly reduced when compared to the baseline. Moreover, both groups showed PAL gains compared to baseline. However, there was no significant difference in mean full-mouth PPD reduction and mean full-mouth PAL gain between non-smokers and smokers. Also, the proportion of sites with PPD $\geq 5.0 \mathrm{~mm}$ was significantly reduced after the non-surgical periodontal therapy in both smokers and non-smokers. However at 12 months, smokers showed less favorable results in terms of significantly higher percentage residual pockets (PPD $\geq 5.0 \mathrm{~mm}$ ) than non-smokers (Table 1).

Change of PPD and PAL at initially diseased sites

For the 594 sites with initial PPD $\geq 5.0 \mathrm{~mm}$, the mean PPD at these initially diseased sites in smokers was $5.85 \pm 0.48 \mathrm{~mm}$ and in non-smokers was $5.94 \pm 0.47$ mm. Both smokers and non-smokers showed significant reductions of probing pocket depth at 3, 6 and 12 months when compared to baseline $(p<0.001)$ (Table 1). In smokers, the PPD at initially diseased sites reduced from $5.85 \pm 0.48 \mathrm{~mm}$ at baseline to $3.00 \pm 0.80 \mathrm{~mm}$ at 12 months. In non-smokers, the corresponding PPD change was from $5.94 \pm 0.47 \mathrm{~mm}$ at baseline to $2.49 \pm 0.50 \mathrm{~mm}$ at 12 months (Table 1). When comparing the two groups, non-smokers showed significantly 
greater PPD reduction at 6 and 12 months $(p<0.01)$ (Fig.1).

The change in PAL at initially diseased sites of the two groups is shown in Fig 2. No significant difference between smokers and non-smokers was detected at any time point.

\section{Multilevel statistical analysis}

Change of PPD at all sites

Altogether, 5814 sites distributed on 969 teeth in these 40 subjects were included for the analysis of reduction in PPD at 3, 6 and 12 months.

The overall mean reductions in PPD at 3, 6 and 12 months were $0.85 \mathrm{~mm}, 0.95$ $\mathrm{mm}$ and $1.00 \mathrm{~mm}$ respectively (Table 2). The Variance Component models showed that significant variations existed at all three levels of the multilevel structure (all 95\% confidence intervals did not cover the value of 0 ). Site-level variation contributed about $80 \%$ of the total variation in reduction in PPD at 3, 6 and 12 months.

Ten independent variables were included in the multilevel multiple regression and the random intercept models with significant variables only are shown in Table 3. The intercept in the model for the reduction in PPD 3-month was $0.62 \mathrm{~mm}$. This indicates that the mean reduction in PPD at 3 months was $0.62 \mathrm{~mm}$ for buccal sites from lower anterior teeth with absence of plaque and BOP at baseline in smokers with mean age of 45.58 years, with a mean 3.78 missing teeth and a mean $63.89 \%$ sites with BOP and $77.11 \%$ with plaque at baseline.

From the random intercept models for all sites there was no statistically significant difference in PPD reduction between non-smokers and smokers throughout the study period $(\mathbf{p}<0.05)$.

Consistently, sites on incisors and canines, on lingual aspects, sites with 
presence of plaque and BOP at baseline, as well as sites from subjects with higher percentages of sites with BOP showed significantly greater reduction in PPD at 3, 6 and 12 months.

The variances at each level were reduced by the inclusion of the ten variables. The total variances of the models were reduced by $7 \%, 8 \%$ and $9 \%$ respectively for reduction in PPD at 3, 6 and 12 months when compared to the corresponding Variance Components models.

\section{Change of PAL at all sites}

Again, 5814 sites distributed on 969 teeth in all the 40 subjects were included for the analyses of gain in PAL at 3, 6 and 12 months.

The overall mean gains in PAL at 3, 6 and 12 months were $0.24 \mathrm{~mm}, 0.30 \mathrm{~mm}$ and $0.37 \mathrm{~mm}$ respectively (Table 2). The Variance Component models showed that significant variations existed at all three levels of the multilevel structure (all 95\% confidence intervals did not cover the value of 0 ) except for the tooth-level at 12 months. Site-level variation contributed from $80 \%$ to $90 \%$ of the total variation in gain in PAL at 3, 6 and 12 months.

From the regression models (Table 3), it was found that there was no significant difference in the gain in PAL at 3, 6 and 12 months between the smokers and non-smokers. Consistently, sites on lingual surfaces showed significantly greater gains in PAL at 3, 6 and 12 months $(p<0.001)$. Moreover, sites on anterior teeth showed slightly greater PAL gain at 6 and 12 months $(p$ $<\mathbf{0 . 0 0 1 )}$.

The variations at the three levels were reduced by $0-30 \%$ with the inclusion of the ten variables. The total variances of the models were reduced only by $2-4 \%$ for the gain in PAL at 3, 6 and 12 months when compared to the corresponding 
Variance Components models.

\section{Change in PPD at initially diseased sites}

Altogether, 594 sites with initial PPD $\geq 5 \mathrm{~mm}$, distributed on 324 teeth in these 40 subjects were included for the analyses of reduction in PPD of initially diseased sites at 3, 6 and 12-months.

The overall mean reductions in PPD of initially diseased sites at 3, 6 and 12 months were $2.55 \mathrm{~mm}, 2.77 \mathrm{~mm}$ and $3.16 \mathrm{~mm}$ respectively (Table 4). The Variance Component models showed that significant variations existed at all three levels of the multilevel structure (all 95\% confidence intervals did not cover the value of 0 ) except for subject level at 3-month. Site-level variation contributed about $70 \%$ to $80 \%$ of the total variation in reduction in PPD at 3,6 and 12 months.

Similar to the analysis for all sites, 10 independent variables were included in the multilevel multiple regression, and the result of random intercept models are shown in Table 5. From the regression models, initially diseased sites of non-smokers consistently showed greater PPD reduction at 3, 6 and 12 months $(0.41 \mathrm{~mm}, 0.79 \mathrm{~mm}$ and $0.68 \mathrm{~mm}$ respectively, $p<0.05)$

In accordance with analysis of all sites, initially diseased sites from anterior teeth were found to have undergone significantly greater reduction in PPD at 3, 6 and 12 months $(p<0.05)$. Contrary to the results of the analysis of all sites, initially diseased sites on lingual aspects with presence of plaque at baseline showed less PPD reduction at 3, 6 and 12 months $(p<0.05)$.

In the analysis for the initially diseased sites, the total variances of the models were reduced by only 9-13\% respectively for reduction in PPD at 3, 6 and 12 months when compared to the corresponding Variance Components models. 


\section{Change in PAL at initially diseased sites}

Those 594 initially diseased sites on 324 teeth in the 40 patients were included for the analyses of gain in PAL at 3, 6 and 12 months.

From the Variance Component models, the overall mean gains in PAL at 3, 6 and 12 months were $0.80 \mathrm{~mm}, 0.83 \mathrm{~mm}$ and $1.21 \mathrm{~mm}$ respectively (Table 4). Significant variations existed at tooth and sites levels but not subject level of the multilevel structure (all 95\% confidence intervals did not cover the value of 0 ) at 3,6 and 12 months. Site-level variation contributed most of the variation in gain in PAL at 3, 6 and 12 months, ranging from $75 \%$ to $80 \%$.

After the inclusion of the $\mathbf{1 0}$ variables, the total variances of the models were reduced by 2-5\% for the gain in PAL at 3, 6 and 12 months when compared to the corresponding Variance Components models.

From the regression models (Table 5), it was found that there was no significant difference between the smokers and non-smokers in the gain in PAL at 3, 6 and 12 months for initially diseased sites $(p>0.05)$. Only subjects with higher percentage of sites with plaque at baseline showed slightly less PAL gain at 12 months $(p<0.05)$.

For tooth level variables, only tooth position showed a significant effect on gain in PAL of initially diseased sites at 6 months. Sites from anterior teeth had significantly greater gain in PAL than sites on posterior teeth at 6 months ( $p$ $<0.05)$.

For the site level, it was found that only sites with absence of plaque at baseline showed greater PAL gain at 3 and 6 months $(p<0.05)$, while the effects of other variables were insignificant. 


\section{Discussion}

Previous studies have generally demonstrated that smokers have increased risk of periodontal destruction and less favorable healing in response to non-surgical periodontal therapy (Preber \& Bergstrom 1986, Preber et al. 1995, Renvert et al. 1998, Jin et al. 2000). However, the factors affecting the variability of treatment outcomes among different smoking patients and at different sites within individual smokers are still not fully understood.

In much periodontal research statistical methods have been applied which generally ignore the fact that many observations are correlated, by combining all site observations into a mean value. Site level observations are not truly independent (Hujoel et al. 1990). Sites are clustered around a tooth and teeth are clustered in individuals. It is therefore, inappropriate to analyse the site-level or subject level observations using single-level, univariate statistical methods since the correlation among sites and/or teeth within an individual invalidates these statistical methods. Consequently, statistical analysis with assumption that the sites observations are independent would generate potentially misleading results (Tu et al., 2004).

Consequently, statistical analysis undertaken on the assumption that site observations are independent could generate potentially misleading interpretations of results (Tu et al. 2004).

A recent study employed a multilevel approach to investigate factors affecting the probability of "pocket closure" for diseased sites 3 months after two separate regimes of non-surgical periodontal therapy (Tomasi et al. 2007). However "pocket closure" is not the only healing response to non-surgical therapy. Therefore, the present study aimed, using multilevel modeling analysis, to investigate the possible factors affecting response of non-surgical periodontal therapy in male Chinese smokers and 
non-smokers in terms of both PPD reduction and PAL gain.

In the present study, results generated from traditional, routine statistical analysis are also presented. It was found that smokers showed less favorable responses after non-surgical therapy. At 12 months, smokers presented with a significantly higher percentage of residual pockets (Table 1). Additionally, smokers showed less PPD reduction in sites with initial PPD $\geq 5 \mathrm{~mm}$ (Fig. 1). However, there was no statistically significant difference in the gain in PAL in initially diseased sites between smokers and non-smokers (Fig. 2). This is in agreement of a recent systematic review concerning effect of smoking on non-surgical therapy (Labriola et al. 2005), although a review of clinical evidence suggests that the majority of studies do show that clinical attachment gain in response to periodontal therapy is impaired in smokers (Heasman et al. 2006).

In order to account for the natural hierarchical structure of periodontal disease measurements, the present study adopted multilevel multiple regressions to analyze reductions in PPD and gains in PAL compared to baseline at 3, 6 and 12 months following non-surgical periodontal therapy. The Variance Component models of our study clearly showed that significant variation existed at most of the levels in the hierarchical structure at all time points (Tables 2 and 4). This indicates that subject, tooth and site level factors are all responsible for the outcome variations of PPD reduction and change in PAL in response to non-surgical periodontal therapy. In addition, this once more demonstrated that analysis which ignores the natural hierarchical structure of periodontal data might provide some inaccurate results. However, this is still a common data management approach in contemporary periodontal research.

The advantage of a multilevel approach can be identified in the difference between routine subject level analysis, shown in Table 1, and the multilevel regression result, shown in Table 5. Routine univariate statistical analysis showed the 
difference of PPD reduction in initially diseased sites smokers and non-smokers to be significant only at 6 months $(p<0.0017)$ and marginally insignificant in 12-months $(p=0.008)$. On the other hand, the multilevel regression for initially diseased sites (Table 5) showed that sites from non-smokers achieved a significantly greater PPD reduction throughout the study period.

Tables 2 and 4 demonstrate that the site level factors contributed around 70 to $80 \%$ of the total variance in healing outcomes, whereas tooth and subject levels only contributed the remaining $20 \%$ to $30 \%$. This implies that most of the variations in outcomes to non-surgical periodontal therapy level result from factors acting at the site level. This is in agreement with a recent study also assessing the relative contribution of multilevel variation for the outcome of subgingival debridement (D'Aiuto et al. 2005) and with a report on both non-surgical and surgical therapy in single-rooted teeth (Kim et al. 2007), both of which found that site level factors had a much greater impact than subject level factors. Indeed, if tooth loss or tooth retention is the true outcome measure of significance after periodontal therapy, it is worth noting that tooth level factors have been shown to be more important than subject level factors in an analysis which factored in tooth and patient level features (Muzzi et al. 2006).

In the multilevel multiple regression models (Tables 3 and 5), 10 independent variables were included. The percentage reduction in variance compared to Variance Component models indicates the amount of variation that could be explained by the 10 independent variables introduced. For PPD reduction, the independent variables used in the present study achieved about $10 \%$ reduction in variance at the 3-, 6- and 12-month re-examinations. Some variables such as presence of BOP at baseline and mean percentage of sites with BOP at baseline seem only to influence the variance for PPD reduction in general for all sites but do not influence the PPD reduction of initially diseased sites, which mostly 
exhibited BOP at baseline.

Only 2-5\% of variance reductions were obtained for gain in PAL in all sites and in initially diseased sites using the same 10 independent variables (Table $3 \&$ 5). It is rational to presume that factors affecting PPD reduction in response to non-surgical periodontal therapy are different from those influencing PAL gain. Further study involving further independent variables is warranted for investigating the factors affecting gain in PAL after non-surgical periodontal therapy.

By means of multilevel modeling analysis, apart from analyzing which variables significantly affect the results of non-surgical periodontal therapy, an understanding of the effects of these individual factors can be generated. In the regression model, utilizing data from 5814 sites of 969 teeth from 40 subjects for all sites (Table 3), sites on anterior teeth, sites with presence of plaque and BOP at baseline, sites on lingual aspects and sites from subjects with higher full-mouth mean BOP\% consistently showed greater PPD reduction.

From Table 3, it appears that the effect of percentage of sites with BOP at baseline on PPD reduction is clinically insignificant $(0.01 \mathrm{~mm})$. However, if a subject's baseline $\mathrm{BOP} \%$ were to be increased by $1 \%$, the PPD reduction of sites in that subject would have been $0.01 \mathrm{~mm}$ greater. Hence if a subject presents with $50 \%$ higher BOP\% at baseline, the PPD reduction of sites in that subject would be all $0.5 \mathrm{~mm}$ greater. Hence greater reductions in PPD can be expected in those presenting with poorer plaque control, and this may be of clinical importance.

It is generally believed that deeper initial pockets show more PPD reduction. However, researchers have questioned whether that correlation of PPD reduction and baseline PPP measurement may only due to "mathematical coupling" (Tu et al., 2002 and Tu et al., 2005). Since the objective of the present study was not testing the relationship between change and initial value of PPD and PAL but 
focusing on the effect of smoking on response after non-surgical periodontal therapy in terms of PPD reduction and PAL gain, the independent variables such as initial PPD and PAL at baseline and full-mouth mean PPD and PAL at baseline were not included in the analysis (Tu et al. 2004). Other multilevel analysis strategies for investigating the relationship between change and initial values are available to address this issue (Blance et al. 2005, Tu et al. 2005, Tu \& Gilthorpe, 2007).

In treating patients with chronic periodontitis, it may be important to focus attention on the response of diseased sites with periodontal pockets rather than gingivitis sites or healthy sites with no increases in PPD. In the present study, a separate set of multilevel multiple regressions was performed to investigate the effects of variables on PPD reduction and PAL gain in sites with baseline PPD $\geq 5 \mathrm{~mm}$. Non-smokers showed consistently greater PPD reduction at initially diseased sites throughout the study (Table 5). The differences were $0.41 \mathrm{~mm}, \mathbf{0 . 7 9} \mathrm{mm}$ and 0.68 mm at the 3-, 6- and 12-month recalls. These results are in agreement with a previous study demonstrating that smokers from the same population have generally less favorable PPD reduction post-treatment (Jin et al. 2000) and implies that the effect of smoking is to reduce the PPD reduction in sites with baseline PPD $\geq 5 \mathrm{~mm}$ by $\mathbf{0 . 4 1}$ $\mathbf{m m}, \mathbf{0 . 7 9} \mathbf{~ m m}$ and $\mathbf{0 . 6 8} \mathbf{~ m m}$ at 3, 6 and 12 months post-therapy respectively. However, it is important to note that the smoking status as a subject level variable was considered in dichotomous fashion, i.e. if the patient is a current smoker or a never smoker. Future studies could include a quantitative measurement such as pack-years and also include former smokers in investigating any dose-related or residual effect of cigarette smoking on periodontal healing.

In addition, initially diseased sites from anterior teeth, diseased sites with absence of plaque at baseline were found to undergo greater PPD reduction throughout the 
course of the study in response to non-surgical periodontal therapy.

In the present study, we have applied the multilevel statistical analysis of the periodontal data derived from investigating treatment responses after non-surgical therapy in smokers and non-smokers. This approach has yielded new insights into and better understanding of the result of non-surgical periodontal treatment and has allowed a comparison of the treatment responses in Chinese male smokers and non-smokers.

\section{Conclusion}

The present study adds to the evidence that smokers generally show less favorable responses after non-surgical mechanical periodontal therapy in terms of pocket depth reduction. Utilizing multilevel modeling enabled an appreciation of the impact of tooth position and site level factors on healing responses to non-surgical periodontal therapy in both smokers and non-smokers. Most of the variations were found to be associated with site level variables. On the basis of this study future studies with larger sample sizes and focusing on different site level variables are warranted. 


\section{Reference}

Albandar, J.M. \& Goldstein, H. (1992) Multi-level statistical models in studies of periodontal diseases. Journal of Periodontology 63, 690-695.

Apatzidou, D.A., Riggio, M.P. \& Kinane, D.F. (2005) Impact of smoking on the clinical, microbiological and immunological parameters of adult patients with periodontitis. Journal of Clinical Periodontology 32, 973-983.

Baljoon, M., Natto, S.\& Bergström, J. (2005) Long-term effect of smoking on vertical periodontal bone loss. Journal of Clinical Periodontology 32, 789-797.

Bergström, J. (2004) Influence of tobacco smoking on periodontal bone height. Long-term observations and a hypothesis. Journal of Clinical Periodontology 31, 260-266.

Bergström, J. \& Boström, L. (2001) Tobacco smoking and periodontal hemorrhagic responsiveness. Journal of Clinical Periodontology 28, 680-685.

Blance, A., Tu, Y.K. \& Gilthorpe, M.S. (2005) A multilevel modelling solution to mathematical coupling. Statistical Methods in Medical Research 14, 553-65.

Boström, L., Linder, L.E. \& Bergström J. (1998) Clinical expression of TNF-alpha in smoking-associated periodontal disease. Journal of Clinical Periodontology 25, $767-773$.

Boström, L., Linder, L.E. \& Bergström J. (1999) Smoking and cervicular fluid levels of IL-6 and TNF-alpha in periodontal disease. Journal of Clinical Periodontology 26, 352-357.

Clarke, N.G., Shephard, B.C. \& Hirsch, R.S. (1981) The effects of intra-arterial epinephrine and nicotine on gingival circulation. Oral Surgery, Oral Medicine, Oral Pathology 52, 577-582.

D'Aiuto, F., Ready, D., Parkar, M. \& Tonetti, M.S. (2005) Relative contribution of patient-, tooth-, and site-associated variability on the clinical outcomes of 
subgingival debridement. I. Probing depths. Journal of Periodontology 76, 398-405.

Giannopoulou, C., Kamma, J.J. \& Mombelli, A. (2003) Effect of inflammation, smoking and stress on gingival crevicular fluid cytokine level. Journal of Clinical Periodontology 30, 145-153.

Gilthorpe, M.S., Griffiths, G.S., Maddick, I.H. \& Zamzuri, A.T. (2000a) The application of multilevel modelling to periodontal research data. Community Dental Health 17, 227-235.

Gilthorpe, M.S., Griffiths, G.S., Maddick, I.H. \& Zamzuri, A.T. (2001) An application of multilevel modelling to longitudinal periodontal research data. Community Dental Health 18, 79-86.

Gilthorpe, M.S., Maddick, I.H. \& Petrie, A. (2000b) Introduction to multilevel modelling in dental research. Community Dental Health 17, 222-226.

Grossi, S.G., Genco, R.J., Machtei, E.E., Ho, A.W., Koch, G., Dunford, R., Zambon, J.J. \& Hausmann, E. (1995) Assessment of risk for periodontal disease. II. Risk indicators for alveolar bone loss. Journal of Periodontology 66, 23-29.

Grossi, S.G., Zambon, J.J., Ho, A.W., Koch, G., Dunford, R.G., Machtei, E.E., Norderyd, O.M. \& Genco, R.J. (1994) Assessment of risk for periodontal disease. I. Risk indicators for attachment loss. Journal of Periodontology 65, 260-267.

Güntsch, A., Erler, M., Preshaw, P.M., Sigusch, B.W., Klinger, G. \& Glockmann, E. (2006) Effect of smoking on crevicular polymorphonuclear neutrophil function in periodontally healthy subjects. Journal of Periodontal Research 41, 184-188.

Haffajee, A.D. \& Socransky, S.S. (2001a) Relationship of cigarette smoking to attachment level profiles. Journal of Clinical Periodontology 28, 283-295.

Haffajee, A.D. \& Socransky, S.S. (2001b) Relationship of cigarette smoking to the 
subgingival microbiota. Journal of Clinical Periodontology 28, 377-388.

Heasman, L., Stacey, F., Preshaw, P.M., McCracken, G.I., Hepburn, S. \& Heasman, P.A. (2006) The effect of smoking on periodontal treatment response: a review of clinical evidence. Journal of Clinical Periodontology 33, 241-253.

Hujoel, P.P., Loesche, W.J. \& DeRouen, T.A. (1990) Assessment of relationships between site-specific variables. Journal of Periodontology 61, 368-372.

Jin, L., Wong, K.Y., Leung, W.K. \& Corbet, E.F. (2000) Comparison of treatment response patterns following scaling and root planing in smokers and non-smokers with untreated adult periodontitis. Journal of Clinical Dentistry 11, $35-41$.

Johnson, J.D., Houchens, D.P., Kluwe, W.M., Craig, D.K. \& Fisher, G.L. (1990) Effects of mainstream and environmental tobacco smoke on the immune system in animals and humans: a review. Critical Reviews in Toxicology 20, 369-395.

Kaldahl, W.B., Kalkwarf, K.L., Patil, K.D., Dyer, J.K. \& Bates, R.E. Jr. (1988) Evaluation of four modalities of periodontal therapy. Mean probing depth, probing attachment level and recession changes. Journal of Periodontology 59; 783-793.

Kim, T.-S., Schenk, A., Lungeanu, D., Reitmeir, P. \& Eickholz P. (2007) Nonsurgical and surgical periodontal therapy in single-rooted teeth. Clinical Oral Investigations 11, 391-399.

Kraal, J.H. \& Kenney E.B. (1979) The response of polymorphonuclear leukocytes to chemotactic stimulation for smokers and non-smokers. Journal of Periodontal Research 14, 383-389. 
Labriola, A., Needleman, I. \& Moles, D.R. (2005) Systematic review of the effect of smoking on non-surgical periodontal therapy. Periodontology $200037,124-137$.

Leung, W. K., Ng, D.K.C., Jin, L. \& Corbet, E.F. (2006) Tooth loss in treated periodontitis patients responsible for their supportive care arrangements. Journal of Clinical Periodontology 33, 265-275.

Lie, M.A., Timmerman, M.F., van der Velden, U. \& van der Weijden, G.A. (1998) Evaluation of 2 methods to assess gingival bleeding in smokers and non-smokers in natural and experimental gingivitis. Journal of Clinical Periodontology 25, 695-700.

Lindhe, J., Socransky, S.S., Nyman, S. \& Westfelt, E. (1987) Dimensional alteration of the periodontal tissues following therapy. International Journal of Periodontics and Restorative Dentistry 7(2), 9-21.

Mariggio, M.A., Guida, L., Laforgia, A., Santacroce, R., Curci, E., Montemurro, P. \& Fumarulo, R. (2001) Nicotine effects on polymorphonuclear cell apoptosis and lipopolysaccharide-induced monocyte functions. A possible role in periodontal disease? Journal of Periodontal Research 36, 32-39.

Matuliene, G., Pjetursson, B.E., Salvi, G.E., Schmidlin, K., Brägger, U., Zwahlen, M. \& Lang, N.P. (2008) Influence of residual pockets on progression of periodontitis and tooth loss: results after 11 years of maintenance. Journal of Clinical Periodontology 35, 685-695.

Mirbod, S.M., Ahing, S.I. \& Pruthi, V.K. (2001) Immunohistochemical study of vestibular gingival blood vessel density and internal circumference in smokers and non-smokers. Journal of Periodontology 72, 1318-1323.

Mooney, J., Hodge, P.J. \& Kinane, D.F. (2001) Humoral immune response in early-onset periodontitis: influence of smoking. Journal of Periodontal Research 
36, 227-232.

Muzzi, L., Nieri, M., Cattabriga, M., Rotundo, R., Cairo, F. \& Pini-Prato, G.P. (2006) The potential prognostic value of some periodontal factors for tooth loss: a retrospective multilevel analysis on periodontal patients treated and maintained over 10 years. Journal of Periodontology 77, 2084-2089.

Ng, S.K. \& Leung, W.K. (2006) A community study on the relationship between stress, coping, affective dispositions and periodontal attachment loss. Community Dentistry and Oral Epidemiology 34, 252-266.

Page, R.C. \& Kornman, K.S. (1997) The pathogenesis of human periodontitis: an introduction. Periodontology 2000 14, 9-11.

Preber, H \& Bergström, J. (1986) The effect of non-surgical treatment on periodontal pockets in smokers and non-smokers. Journal of Clinical Periodontology 13, $319-323$.

Preber, H., Linder, L. \& Bergström, J. (1995) Periodontal healing and periopathogenic microflora in smokers and non-smokers. Journal of Clinical Periodontology 22, $946-952$.

Ramfjord, S.P., Caffesse, R.G., Morrison, E.C., Hill, R.W., Kerry, G.J., Appleberry, E.A., Nissle, R.R. \& Stults, D.L. (1987) 4 modalities of periodontal treatment compared over 5 years. Journal of Clinical Periodontology 14; 445-452.

Rasbash, J., Browne, W., Goldstein, H., Yang, M., Plewis, I., Healy, M., Woodhouse, G., Draper, D., Langford, I. \& Lewis, T. (2000). A user's guide to MLwiN. Version 2.1. Multilevel Models Project, Institute of Education, University of London.

Raulin, L.A., McPherson, J.C. 3rd, McQuade, M.J. \& Hanson, B.S. (1998) The effect of nicotine on the attachment of human fibroblasts to glass and human root surfaces in vitro. Journal of Periodontology 59, 318-325. 
Renvert, S., Dahlén, G. \& Wikström, M. (1998) The clinical and microbiological effects of non-surgical periodontal therapy in smokers and non-smokers. Journal of Clinical Periodontology 25, 153-157.

Sanz, M. \& Teughels, W. (2008) Innovations in non-surgical periodontal therapy: Consensus Report of the Sixth European Workshop on Periodontology. Journal of Clinical Periodontology 35(s8), 3-7.

Susin, C., Oppermann, R.V., Haugejorden, O. \& Albandar, J.M. (2004) Periodontal attachment loss attributable to cigarette smoking in an urban Brazilian population. Journal of Clinical Periodontology 31, 951-958.

Tomasi, C., Leyland, A.H. \& Wennström, J.L. (2007) Factors influencing the outcome of non-surgical periodontal treatment: a multilevel approach. Journal of Clinical Periodontology 34, 682-690.

Tong, K.S., Zee, K.-Y., Lee, D.H. \& Corbet, E.F. (2003) Clinical responses to mechanical periodontal treatment in Chinese chronic periodontitis patients with and without Actinobacillus actinomycetemcomitans. Journal of Periodontology 74, 1582-1588.

Tu, Y.K. \& Gilthorpe, M.S. (2007) Revisiting the relation between change and initial value: A review and evaluation. Statistics in Medicine 26, 443-457.

Tu, Y.K, Baelum, V. \& Gilthorpe, M.S. (2005) The problem of analysing the relationship between change and initial value in oral health research. European Journal of Oral Sciences. 113, 271-8.

Tu, Y.K., Gilthorpe, M.S. \& Griffiths, G.S. (2002) Is reduction of pocket probing depth correlated with the baseline value or is it "mathematical coupling"? . Journal of Dental Research 81, 722-726.

Tu, Y.K, Gilthorpe, M.S., Griffiths, G.S., Maddick, I.H., Eaton, K.A. \& Johnson, N.W. (2004) The application of multilevel modeling in the analysis of longitudinal 
periodontal data--part II: changes in disease levels over time. Journal of Periodontology 75, 137-45.

van Winkelhoff, A.J., Bosch-Tijhof, C.J., Winkel, E.G. \& van der Reijden, W.A. (2001) Smoking affects the subgingival microflora in periodontitis. Journal of Periodontology 72, 666-671. 


\section{Legend}

Fig. 1. Change in probing pocket depth $(\mathrm{PPD} ; \pm \mathrm{SD})$ of sites with $\mathrm{PPD} \geq 5.0 \mathrm{~mm}$ at baseline. * Statistically significant differences between groups after adjustment for multiple comparisons $(p<0.001)$.

Fig. 2. Change in PAL $( \pm \mathrm{SD})$ of sites with $\mathrm{PPD} \geq 5.0 \mathrm{~mm}$ at baseline. 


\section{Clinical Relevance}

Scientific rationale: It would be useful for clinicians to able to predict outcomes of non-surgical mechanical periodontal therapy based on clinical data. The hierarchical structure of periodontal disease measurements, sites' measurements clustered around teeth and then teeth clustered within individuals applies to periodontal disease clinical findings and to outcomes of periodontal therapy, hence multilevel analysis approach is adopted in this study. Practical implications: Multilevel analysis revealed that for diseased sites without plaque at baseline, from anterior teeth, in non-smokers were found to response favorably throughout 12 month post-treatment. Such analysis strategy could be applied to other periodontal treatment modalities. 
Table 1. Subject level clinical parameters over study period

\begin{tabular}{|c|c|c|c|c|c|c|c|c|}
\hline & \multicolumn{4}{|c|}{ Non-smokers $(\mathrm{n}=20)$} & \multicolumn{4}{|c|}{ Smokers $(n=20)$} \\
\hline & \multirow[b]{2}{*}{ Baseline } & \multicolumn{3}{|c|}{ Months post-treatment } & \multirow[b]{2}{*}{ Baseline } & \multicolumn{3}{|c|}{ Months post-treatment } \\
\hline & & 3 & 6 & 12 & & 3 & 6 & 12 \\
\hline Full mouth plaque $\%$ & $75.45 \pm 14.95$ & $40.70 \pm 17.21$ & $32.81 \pm 17.21$ & $26.55 \pm 14.19$ & $77.36 \pm 10.96$ & $35.21 \pm 23.50$ & $26.36 \pm 13.61$ & $33.79 \pm 15.07$ \\
\hline Full mouth BOP \% & $73.45 \pm 21.02$ & $42.01 \pm 15.53$ & $37.95 \pm 15.40$ & $24.92 \pm 10.44$ & $54.32 \pm 13.68$ & $32.04 \pm 11.73$ & $23.97 \pm 9.65$ & $26.91 \pm 10.85$ \\
\hline Full mouth mean PPD (mm) & $2.82 \pm 0.73$ & $1.95 \pm 0.42$ & $1.82 \pm 0.31$ & $1.71 \pm 0.28$ & $2.89 \pm 0.52$ & $2.06 \pm 0.37$ & $1.99 \pm 0.34$ & $2.01 \pm 0.38$ \\
\hline Full mouth mean PAL $(\mathrm{mm})^{*}$ & $3.69 \pm 0.97$ & --- & ---8 & --- & $3.71 \pm 0.68$ & --- & --- & --- \\
\hline PPD reduction (mm) & --- & $0.88 \pm 0.57$ & $1.00 \pm 0.55$ & $1.11 \pm 0.69$ & --- & $0.83 \pm 0.28$ & $0.91 \pm 0.28$ & $0.89 \pm 0.32$ \\
\hline PAL gain (mm) & --- & $0.18 \pm 0.48$ & $0.33 \pm 0.54$ & $0.50 \pm 0.52$ & --- & $0.28 \pm 0.18$ & $0.26 \pm 0.21$ & $0.31 \pm 0.42$ \\
\hline$\%$ of pocket $\geq 5.0 \mathrm{~mm}$ & $11.43 \pm 12.14$ & $1.98 \pm 2.13$ & $1.15 \pm 1.52$ & $0.80 \pm 0.94$ & $9.98 \pm 9.69$ & $2.76 \pm 3.01$ & $2.52 \pm 2.68$ & $3.37 \pm 3.24$ \\
\hline Diseased site mean PPD (mm) & $5.94 \pm 0.47$ & $3.29 \pm 0.57$ & $2.89 \pm 0.39$ & $2.49 \pm 0.50$ & $5.85 \pm 0.48$ & $3.51 \pm 0.71$ & $3.46 \pm 0.54$ & $3.00 \pm 0.80$ \\
\hline Diseased site mean PAL $(\mathrm{mm})^{*}$ & $6.86 \pm 0.88$ & --- & --- & --- & $6.61 \pm 0.64$ & --- & --- & --- \\
\hline Diseased site PPD reduction (mm) & --- & $2.65 \pm 0.66$ & $3.05 \pm 0.61$ & $3.45 \pm 0.62$ & --- & $2.33 \pm 0.50$ & $2.38 \pm 0.57$ & $2.84 \pm 0.75$ \\
\hline Diseased site plaque $\%$ & $86.48 \pm 14.67$ & $65.24 \pm 26.59$ & $50.22 \pm 26.83$ & $42.45 \pm 25.57$ & $92.53 \pm 10.24$ & $53.23 \pm 29.31$ & $45.70 \pm 23.07$ & $58.27 \pm 21.86$ \\
\hline Diseased site $\mathrm{BOP} \%$ & $90.25 \pm 15.86$ & $65.81 \pm 21.94$ & $55.74 \pm 19.73$ & $35.86 \pm 22.49$ & $71.89 \pm 19.54$ & $44.92 \pm 22.47$ & $36.68 \pm 20.88$ & $42.42 \pm 21.54$ \\
\hline
\end{tabular}

Bold fonts: Statistically significance between groups regarding data at baseline $(p<0.05)$

Bold and italic fonts: Statistically significance between groups after adjustment for multiple comparison $(p<0.0017)$

*Measured manually by PCP-UNC 15, Hu-Friedy probe, Chicago, IL (Cheng et al., 2008); all other measurements of PPD and PAL used Florida Probe® 
Table 2. Variance Components models for reduction in PPD and gain in PAL for all sites

\begin{tabular}{|c|c|c|c|c|c|c|}
\hline & \multicolumn{3}{|c|}{ Reduction in PPD } & \multicolumn{3}{|c|}{ Gain in PAL } \\
\hline & 3-month & 6-month & 12-month & 3-month & 6-month & 12-month \\
\hline Mean (intercept) & $\begin{array}{c}0.85 \\
(0.72,0.99)\end{array}$ & $\begin{array}{c}0.95 \\
(0.82,1.08)\end{array}$ & $\begin{array}{c}1.00 \\
(0.83,0.16)\end{array}$ & $\begin{array}{c}0.24 \\
(0.13,0.34)\end{array}$ & $\begin{array}{c}0.30 \\
(0.17,0.42)\end{array}$ & $\begin{array}{c}0.37 \\
(0.23,0.51)\end{array}$ \\
\hline \multicolumn{7}{|l|}{ Variance } \\
\hline Tooth (level-2) & $\begin{array}{c}0.15 \\
(0.12,0.18)\end{array}$ & $\begin{array}{c}0.14 \\
(0.10,0.17)\end{array}$ & $\begin{array}{c}0.15 \\
(0.11,0.19)\end{array}$ & $\begin{array}{c}0.11 \\
(0.08,0.14)\end{array}$ & $\begin{array}{c}0.13 \\
(0.10,016)\end{array}$ & $\begin{array}{c}0.03 \\
(0.00,0.07)\end{array}$ \\
\hline Site (level-1) & $\begin{array}{c}1.15 \\
(1.10,1.20)\end{array}$ & $\begin{array}{c}1.21 \\
(1.16,1.26)\end{array}$ & $\begin{array}{c}1.53 \\
(1.47,1.59)\end{array}$ & $\begin{array}{c}1.24 \\
(1.19,1.29)\end{array}$ & $\begin{array}{c}1.22 \\
(1.17,1.27)\end{array}$ & $\begin{array}{c}1.89 \\
(1.82,1.97)\end{array}$ \\
\hline \multicolumn{7}{|l|}{$\%$ total variance } \\
\hline Subject (level-3) & 12 & 11 & 14 & 8 & 10 & 9 \\
\hline Tooth (level-2) & 10 & 9 & 8 & 7 & 9 & 1 \\
\hline Site (level-1) & 78 & 80 & 78 & 85 & 81 & 90 \\
\hline
\end{tabular}


Table 3. Random intercept models for reduction in PPD and gain in PAL for all sites

\begin{tabular}{|c|c|c|c|c|c|c|}
\hline \multirow[b]{2}{*}{ Variables } & \multicolumn{3}{|c|}{ Reduction in PPD } & \multicolumn{3}{|c|}{ Gain in PAL } \\
\hline & $\begin{array}{c}\text { 3-month } \\
\text { Estimate (SE) }\end{array}$ & $\begin{array}{c}\text { 6-month } \\
\text { Estimate (SE) }\end{array}$ & $\begin{array}{l}\text { 12-month } \\
\text { Estimate (SE) }\end{array}$ & $\begin{array}{c}\text { 3-month } \\
\text { Estimate (SE) }\end{array}$ & $\begin{array}{c}\text { 6-month } \\
\text { Estimate (SE) }\end{array}$ & $\begin{array}{c}\text { 12-month } \\
\text { Estimate (SE) }\end{array}$ \\
\hline Intercept & $0.62 \pm 0.10$ & $0.66 \pm 0.09$ & $0.60 \pm 0.11$ & $0.13 \pm 0.09$ & $0.14 \pm 0.10$ & $0.22 \pm 0.10$ \\
\hline \multicolumn{7}{|l|}{ Subject-level } \\
\hline Smoking (non-smoker vs smoker) & $-0.11 \pm 0.13$ & $-0.10 \pm 0.12$ & $-0.01 \pm 0.15$ & $-0.15 \pm 0.12$ & $-0.04 \pm 0.14$ & $0.06 \pm 0.14$ \\
\hline Age at baseline & $0.01 \pm 0.01$ & $0.01 \pm 0.01$ & $0.01 \pm 0.01$ & $0.01 \pm 0.01$ & $0.01 \pm 0.01$ & $<0.01 \pm 0.01$ \\
\hline Number of missing teeth & $0.02 \pm 0.02$ & $0.03 \pm 0.02$ & $0.06 \pm 0.03$ & $0.01 \pm 0.02$ & $-0.01 \pm 0.02$ & $<0.01 \pm 0.02$ \\
\hline \multicolumn{7}{|l|}{ Tooth-level } \\
\hline Tooth position (post. vs. ant.) & $-0.10 \pm 0.04$ & $-0.11 \pm 0.04$ & $-0.140 \pm 0.04$ & $<0.01 \pm 0.04$ & $-0.13 \pm 0.04$ & $-0.15 \pm 0.04$ \\
\hline Arch (lower vs. upper) & $-0.06 \pm 0.04$ & $-0.04 \pm 0.04$ & $0.01 \pm 0.04$ & $-0.04 \pm 0.04$ & $0.02 \pm 0.04$ & $0.06 \pm 0.04$ \\
\hline & $-0.11 \pm 0.13$ & $-0.10 \pm 0.12$ & $-0.01 \pm 0.15$ & $-0.15 \pm 0.12$ & $-0.04 \pm 0.14$ & $0.06 \pm 0.14$ \\
\hline Site-level & $0.01 \pm 0.01$ & $0.01 \pm 0.01$ & $0.01 \pm 0.01$ & $0.01 \pm 0.01$ & $0.01 \pm 0.01$ & $<0.01 \pm 0.01$ \\
\hline Presence of plaque at baseline & $0.02 \pm 0.02$ & $0.03 \pm 0.02$ & $0.06 \pm 0.03$ & $0.01 \pm 0.02$ & $-0.01 \pm 0.02$ & $<0.01 \pm 0.02$ \\
\hline \multicolumn{7}{|l|}{ Variance } \\
\hline Subject & 0.11 & 0.10 & 0.14 & 0.10 & 0.13 & 0.13 \\
\hline Tooth & 0.14 & 0.12 & 0.14 & 0.11 & 0.13 & 0.03 \\
\hline Site & 1.12 & 1.17 & 1.49 & 1.22 & 1.20 & 1.87 \\
\hline Total variance & 1.38 & 1.40 & 1.77 & 1.43 & 1.36 & 2.03 \\
\hline \multicolumn{7}{|c|}{$\%$ reduction in variance (compared to Variance Component models in Table 2) } \\
\hline Subject & 34 & 41 & 46 & 11 & 9 & 30 \\
\hline Tooth & 6 & 9 & 9 & 0 & 0 & 0 \\
\hline Site & 2 & 3 & 3 & 1 & 2 & 1 \\
\hline Total variance & 7 & 8 & 9 & 2 & 2 & 4 \\
\hline
\end{tabular}

Bold fonts: $p<0.05$; Bold and italic fonts: $p<0.001$ 
Table 4. Variance Components models for reduction in PPD and gain in PAL for initially diseased sites*

\begin{tabular}{|c|c|c|c|c|c|c|}
\hline & \multicolumn{3}{|c|}{ Reduction in PPD } & \multicolumn{3}{|c|}{ Gain in PAL } \\
\hline & 3-month & 6-month & 12-month & 3-month & 6-month & 12-month \\
\hline Mean (intercept) & $\begin{array}{c}2.55 \\
(2.35,2.74)\end{array}$ & $\begin{array}{c}2.77 \\
(2.55,3.00)\end{array}$ & $\begin{array}{c}3.16 \\
(2.91,3,42)\end{array}$ & $\begin{array}{c}0.80 \\
(0.63,0.97)\end{array}$ & $\begin{array}{c}0.83 \\
(0.62,1.03)\end{array}$ & $\begin{array}{c}1.21 \\
(0.98,1.44)\end{array}$ \\
\hline \multicolumn{7}{|l|}{ Variance } \\
\hline Tooth (level-2) & $\begin{array}{c}0.35 \\
(0.07,0.63)\end{array}$ & $\begin{array}{c}0.38 \\
(0.10,0.67)\end{array}$ & $\begin{array}{c}0.43 \\
(0.14,0.71)\end{array}$ & $\begin{array}{c}0.83 \\
(0.41,1.25)\end{array}$ & $\begin{array}{c}0.39 \\
(0.07,0.70)\end{array}$ & $\begin{array}{c}0.73 \\
(0.28,1.18)\end{array}$ \\
\hline Site (level-1) & $\begin{array}{c}2.07 \\
(1.74,2.39)\end{array}$ & $\begin{array}{c}2.03 \\
(1.71,2.34)\end{array}$ & $\begin{array}{c}1.90 \\
(1.60,2.20)\end{array}$ & $\begin{array}{c}2.55 \\
(2.15,2.96)\end{array}$ & $\begin{array}{c}2.32 \\
(1.96,2.68)\end{array}$ & $\begin{array}{c}3.03 \\
(2.55,3.50)\end{array}$ \\
\hline \multicolumn{7}{|l|}{$\%$ total variance } \\
\hline Subject (level-3) & 6 & 9 & 14 & 0 & 6 & 4 \\
\hline Tooth (level-2) & 14 & 15 & 16 & 25 & 14 & 19 \\
\hline Site (level-1) & 80 & 76 & 70 & 75 & 80 & 77 \\
\hline
\end{tabular}

$95 \%$ confidence intervals in parenthesis.

*Baseline PPD $\geq 5.0 \mathrm{~mm}$ 
Table 5. Final multilevel multiple regression random intercept models for reduction in PPD and gain in PAL for initially diseased sites*

\begin{tabular}{|c|c|c|c|c|c|c|}
\hline \multirow[b]{2}{*}{ Variables } & \multicolumn{3}{|c|}{ Reduction in PPD } & \multicolumn{3}{|c|}{ Gain in PAL } \\
\hline & $\begin{array}{c}\text { 3-month } \\
\text { Estimate (SE) }\end{array}$ & $\begin{array}{c}\text { 6-month } \\
\text { Estimate (SE) }\end{array}$ & $\begin{array}{l}\text { 12-month } \\
\text { Estimate (SE) }\end{array}$ & $\begin{array}{c}\text { 3-month } \\
\text { Estimate (SE) }\end{array}$ & $\begin{array}{c}\text { 6-month } \\
\text { Estimate (SE) }\end{array}$ & $\begin{array}{c}\text { 12-month } \\
\text { Estimate (SE) }\end{array}$ \\
\hline Intercept & $3.45 \pm 0.27$ & $3.26 \pm 0.27$ & $3.65 \pm 0.28$ & $1.71 \pm 0.31$ & $1.49 \pm 0.29$ & $2.12 \pm 0.34$ \\
\hline \multicolumn{7}{|l|}{ Subject-level } \\
\hline Smoking (non-smoker vs smoker) & $0.41 \pm 0.20$ & $0.79 \pm 0.20$ & $0.68 \pm 0.24$ & $-0.19 \pm 0.22$ & $-0.09 \pm 0.22$ & $-0.13 \pm 0.25$ \\
\hline Age at baseline & $0.01 \pm 0.02$ & $0.02 \pm 0.02$ & $<0.01 \pm 0.02$ & $0.02 \pm 0.02$ & $0.02 \pm 0.02$ & $<0.01 \pm 0.02$ \\
\hline Number of missing teeth & $<0.01 \pm 0.03$ & $<0.01 \pm 0.03$ & $0.06 \pm 0.04$ & $-0.02 \pm 0.03$ & $-0.04 \pm 0.04$ & $<0.01 \pm 0.04$ \\
\hline \%of sites with plaque at baseline & $<0.01 \pm<0.01$ & $<0.01 \pm<0.01$ & $-0.02 \pm<0.01$ & $-0.002 \pm<0.01$ & $-0.01 \pm<0.01$ & $-0.02 \pm<0.01$ \\
\hline$\%$ of sites with BOP at baseline & $<0.01 \pm<0.01$ & $<0.01 \pm<0.01$ & $<0.01 \pm<0.01$ & $<0.01 \pm<0.01$ & $<0.01 \pm<0.01$ & $<0.01 \pm<0.01$ \\
\hline \multicolumn{7}{|l|}{ Tooth-level } \\
\hline Tooth position (post. vs. ant.) & $-0.35 \pm 0.15$ & $-0.48 \pm 0.14$ & $-0.35 \pm 0.15$ & $-0.23 \pm 0.18$ & $-0.31 \pm 0.16$ & $-0.36 \pm 0.19$ \\
\hline Arch (lower vs. upper) & $-0.02 \pm 0.14$ & $-0.02 \pm 0.14$ & $0.06 \pm 0.14$ & $-0.25 \pm 0.17$ & $-0.06 \pm 0.15$ & $-0.12 \pm 0.18$ \\
\hline \multicolumn{7}{|l|}{ Site-level } \\
\hline Presence of plaque at baseline & $-0.55 \pm 0.19$ & $-0.44 \pm 0.19$ & $-0.45 \pm 0.19$ & $-0.48 \pm 0.22$ & $-0.45 \pm 0.20$ & $-0.25 \pm 0.24$ \\
\hline Presence of $\mathrm{BOP}$ at baseline & $-0.21 \pm 0.20$ & $0.10 \pm 0.19$ & $-0.16 \pm 0.20$ & $-0.14 \pm 0.23$ & $-0.03 \pm 0.21$ & $-0.41 \pm 0.25$ \\
\hline Surface (lingual vs buccal) & $-0.39 \pm 0.13$ & $-0.42 \pm 0.13$ & $-0.20 \pm 0.13$ & $-0.07 \pm 0.15$ & $<0.01 \pm 0.14$ & $-0.09 \pm 0.16$ \\
\hline \multicolumn{7}{|l|}{ Variance } \\
\hline Subject & 0.11 & 0.05 & 0.16 & 0.00 & 0.07 & 0.06 \\
\hline Tooth & 0.14 & 0.32 & 0.41 & 0.81 & 0.38 & 0.76 \\
\hline Site & 1.12 & 1.94 & 1.85 & 2.50 & 2.30 & 2.97 \\
\hline Total variance & 1.38 & 2.30 & 2.42 & 3.31 & 2.75 & 3.78 \\
\hline \multicolumn{7}{|c|}{$\%$ reduction in variance (compared to Variance Component models in Table 4 ) } \\
\hline Subject & 79 & 80 & 60 & 100 & 57 & 64 \\
\hline Tooth & -8 & 17 & 4 & 3 & 1 & -3 \\
\hline Site & 6 & 4 & 3 & 2 & 1 & 2 \\
\hline Total variance & 9 & 13 & 11 & 2 & 5 & 3 \\
\hline
\end{tabular}

\footnotetext{
Bold fonts: $p<0.05 ;$ Bold and italic fonts: $p<0.001$
}

*Baseline PPD $\geq 5.0 \mathrm{~mm}$ 
Fig. 1. Change in probing pocket depth (PPD; \pm SD) of sites with PPD $\geq 5.0 \mathrm{~mm}$ at baseline. *Statistically significant differences between groups after adjustment for multiple comparisons $(p<0.001)$.

$131 \times 74 \mathrm{~mm}(600 \times 600 \mathrm{DPI})$ 


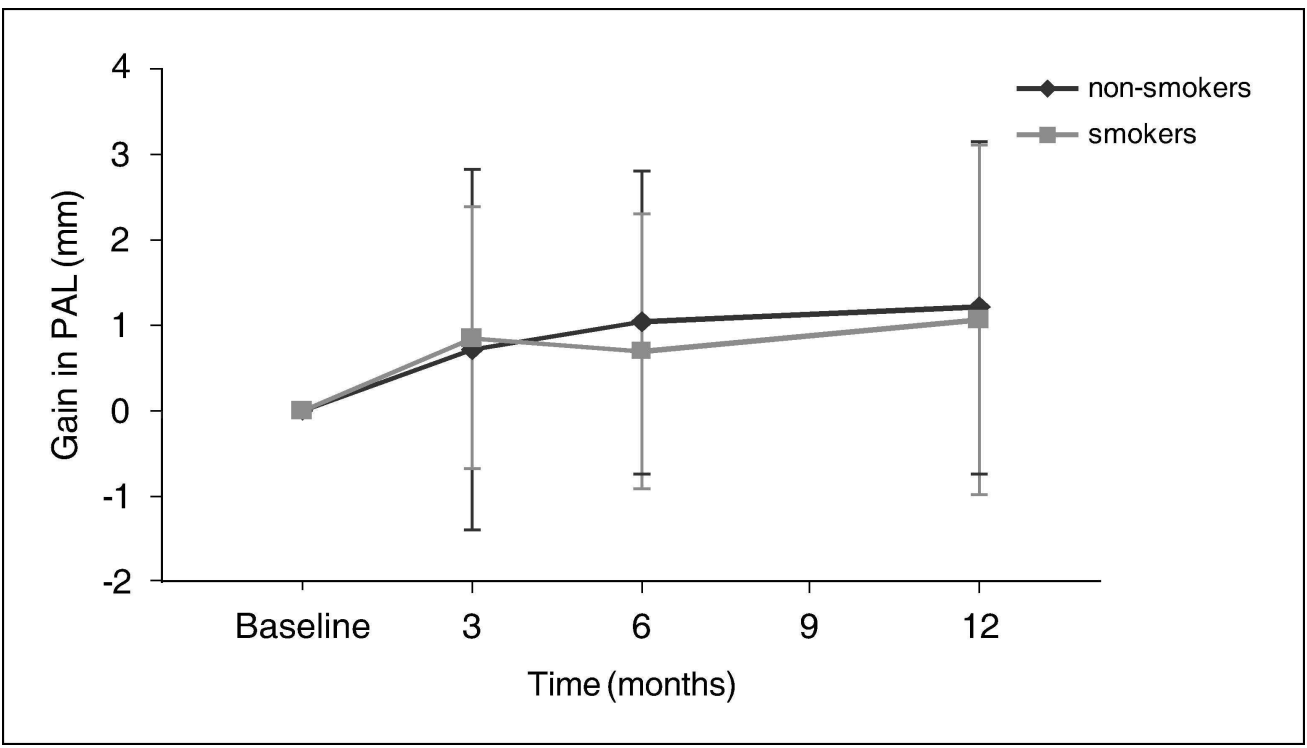

Fig. 2. Change in PAL ( \pm SD) of sites with PPD $\geq 5.0 \mathrm{~mm}$ at baseline. $131 \times 74 \mathrm{~mm}(600 \times 600 \mathrm{DPI})$ 